\title{
MEASUREMENT OF COHERENT OFF-AXIS UNDULATOR RADIATION AS A BEAM DIAGNOSTIC
}

\author{
C. P. Neuman*, W. S. Graves, G. L. Carr, BNL, Upton, NY 11973, USA \\ P. G. O'Shea, University of MD, College Park, MD 20742, USA
}

\section{Abstract}

A diagnostic undulator is used to measure relative electron bunch lengths. The radiation produced by the short undulator is observed off-axis so that the beam is not interrupted. Wavelengths radiated which are longer than the bunch length are coherent and may be more intense than the shorter wavelengths by a factor of up to $10^{9}$. The total energy radiated thus changes as the bunch length changes, and a measurement of the radiated energy provides a robust beam diagnostic. This non-disturbing diagnostic would be useful in applications where the length of short electron bunches is critical, such as singlepass FELs and linear colliders. For example, real-time bunch length measurements could be used to optimize performance of a single-pass FEL. The experiment is performed at the Source Development Lab (SDL) at Brookhaven National Laboratory. The theory and description of the experiment are reviewed, and the first experimental results are presented.

\section{REVIEW OF THEORY}

The theory of coherent off-axis undulator radiation (COUR) has been described [1]. Both a multi-mode approach and a Liénard-Wiechert approach are used to describe the electric field of the radiation produced by the accelerating electrons. It is concluded that the LiénardWiechert approach is the most appropriate and the most useful approach for the case described in this study [2]. A review of the Liénard-Wiechert approach follows.

The electric field is derived from Liénard-Wiechert potentials:

$$
\vec{E}=\frac{q^{2}}{4 \pi \varepsilon_{0}}\left\{\frac{1}{\gamma^{2} R^{2}} \frac{\hat{n}-\vec{\beta}}{(1-\hat{n} \cdot \vec{\beta})^{3}}+\frac{1}{c R} \frac{\hat{n} \times[(\hat{n}-\vec{\beta}) \times \dot{\vec{\beta}}]}{(1-\hat{n} \cdot \vec{\beta})^{3}}\right\} .
$$

$R$ and $\hat{n}$ are the distance and direction, respectively, from the electron to the observation point. The first term, which depends on the electron's velocity, is neglected. The spectral energy is given by:

$$
\frac{d^{2} I}{d \omega d \Omega}=\frac{\varepsilon_{0}}{c \pi} \frac{q^{2}}{\left(4 \pi \varepsilon_{0}\right)^{2}}\left|\int_{0}^{\frac{L}{\bar{\beta}_{c}}} e^{i \omega\left(t+\frac{R}{c}\right)} \frac{\hat{n} \times[(\hat{n}-\vec{\beta}) \times \dot{\vec{\beta}}]}{(1-\hat{n} \cdot \vec{\beta})^{2}} d t\right|^{2} .
$$

A bunch form factor is used to account for the effects of the finite bunch shape. The energy for $N_{e}$ electrons is given by

$$
W_{N_{e} \text { electrons }}=W_{1 \text { electron }}\left[N_{e}+N_{e}\left(N_{e}-1\right) f(\omega)\right],
$$

where $f(\omega)$ is the bunch form factor:

$$
f(\omega)=\left|\iint d y d z S_{y}(y) S_{z}(z) e^{-i \frac{\omega}{c} y \sin \theta+i \frac{\omega}{c} z \cos \theta}\right|^{2} .
$$

$S_{y}(y)$ and $S_{z}(z)$ are the transverse and longitudinal electron bunch densities, respectively. Eqs. 3 and 4 include the coherence effects of the radiation.

A numerical simulation using this method reveals interesting characteristics of off-axis undulator radiation [1]. In particular, the effect of changes in bunch length on the total radiated energy is calculated for both a Gaussianand a top-hat-shaped bunch. The result of the calculation is shown in Fig. 1.

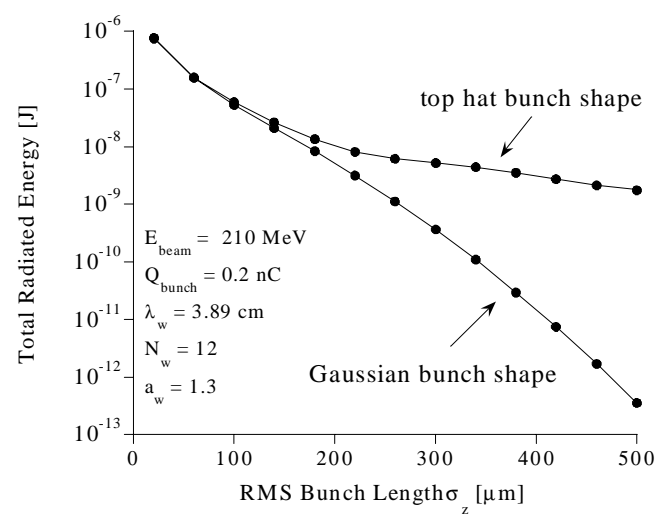

Figure 1: Effect of bunch length on radiated energy.

\section{EXPERIMENTAL APARATUS}

An experiment has been conducted at the Source Development Lab at Brookhaven National Laboratory. The purpose of the experiment is to measure coherent offaxis undulator radiation and to determine its usefulness for measuring electron bunch lengths.

\subsection{The accelerator System}

The SDL accelerator system consists of a photocathode electron gun, which produces electron bunches with up to $1 \mathrm{nC}$ of charge, followed by four $2.856 \mathrm{GHz}$ SLAC-type

\footnotetext{
* Corresponding author. Tel.: +301-405-4977. Email cneuman@phy.duke.edu.
} 
accelerator sections [3]. The electron bunch is compressed in the chicane bunch compressor which is located after the first two accelerator sections (Fig. 2). The compressor compresses the bunch by an amount determined by the phase of the electron on the accelerating wave. Ultrashort bunches, $20 \mu \mathrm{m}$ to $600 \mu \mathrm{m}$ long, are produced. The last two accelerator sections bring the electron bunch to its final energy of $200 \mathrm{MeV}$. The undulator is installed immediately after the last accelerator section.

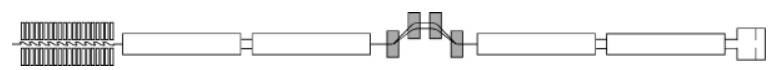

Figure 2: The accelerator system. The electron gun is on the right side, the chicane bunch compressor is in the middle, and an undulator is shown on the left side.

\subsection{The undulator}

The undulator is a 12-pole prototype for the $10 \mathrm{~m}$ NISUS undulator. The magnets are mounted in a configuration that allows the undulator to be removed from the beamline without removing vacuum components. See Fig. 3.

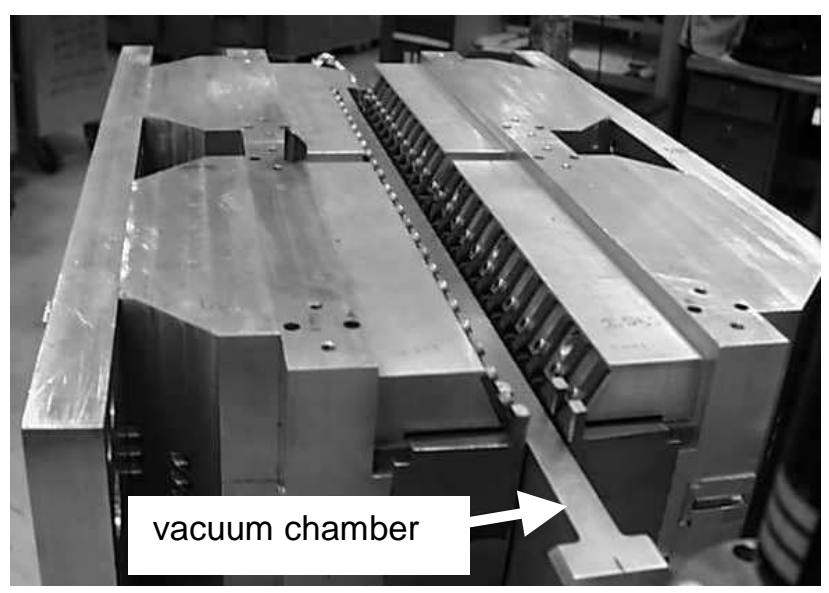

Figure 3: Undulator with the vacuum chamber installed between the magnet poles.

\subsection{Light transport}

The layout of the detection components is seen in Fig. 4. The undulator radiation is reflected into the light pipe by a mirror which can be adjusted by an actuator so that it catches off-axis radiation but does not disturb the electron beam. The radiation exits the beamline through a 1" diameter crystal quartz window with a $0^{\circ}$ crystal orientation. The 1" diameter light pipe acts as a waveguide for the radiation and allows all of the light entering the light pipe to reach the detector.

\subsection{The detector}

The detector is a liquid-helium-cooled bolometer which operates at a temperature of $4.2 \mathrm{~K}$. The detector's wavelength response is $14 \mu \mathrm{m}$ to $1 \mathrm{~mm}$, which is an ideal range for measuring electron bunch lengths from $20 \mu \mathrm{m}$ to
$600 \mu \mathrm{m}$. The bolometer element is silicon and is mounted on a diamond substrate. The low temperature operation reduces noise and allows the detector to detect as little as $1 \mathrm{pJ}$ in $1 \mathrm{~ms}$.

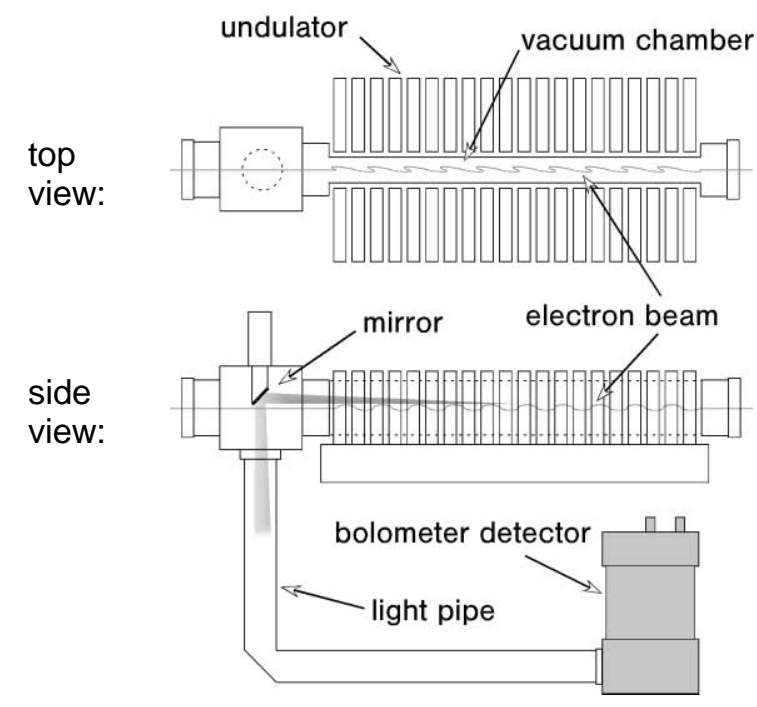

Figure 4: Experimental setup.

\section{EXPERIMENT RESULTS}

Experimental results are shown in Fig. 5. The experimental data is collected by recording the signal level from the detector as the electron phase is varied. The electron phase is the phase of the electron bunch on the accelerating wave before the bunch reaches the chicane bunch compressor. This phase corresponds to the length of bunch length after the chicane as stated above. The phase is converted to bunch length with errors in the conversion indicated by the horizontal error bars. The signal level is converted to the amount of energy in arbitrary units. The signal level fluctuates by approximately plus or minus 10 percent. The results indicate that bunch-length-dependent radiation is observed and that the detected energy behaves in a way that is consistent with theory.

To determine if the radiation is coherent, the laser energy on the cathode is varied in order to change the number of electrons in each bunch. The radiated energy for each cathode laser energy is measured. The results in Fig. 6 show that the signal amplitude, which corresponds to the radiated energy, scales quadratically with the charge of the electron bunch, which corresponds to the number of electrons. This quadratic dependence indicates that the radiation observed is coherent.

At this point, it is determined that coherent bunchlength-dependent radiation is observed. The next step is to demonstrate that the source of the radiation is, in fact, the undulator. When the undulator is removed from the beamline, it is observed that the signal level does not change significantly. However, it is determined that the undulator does produce radiation by observing visible 
radiation in the forward direction. Thus, it is concluded that coherent undulator radiation could be present, although it likely comprises a small fraction of the radiation that is observed. Furthermore, it is assumed that there is another source of radiation which obscures the coherent undulator radiation. A likely candidate for this radiation source is the upstream chicane bunch compressor. The bunch compressor consists of four dipole magnets which are known to produce coherent synchrotron radiation. This radiation could be propagated down the beamline.

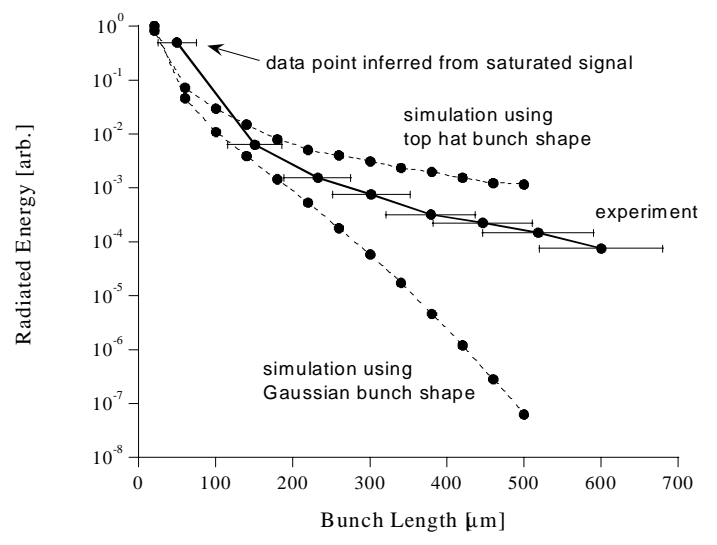

Figure 5: Experimental results (solid) compared with theory (dashed).

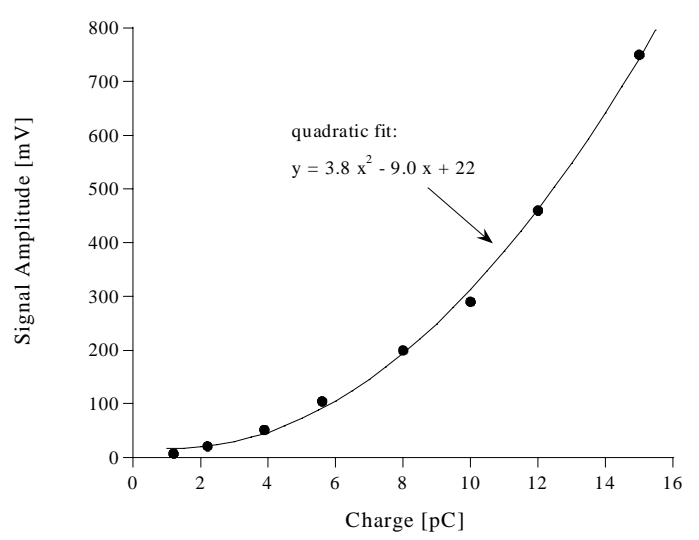

Figure 6: Quadratic dependence of radiated energy on number of electrons in the bunch.

\section{FUTURE WORK}

Future experiments will be performed to determine the source of the radiation that is observed and to isolate the undulator radiation, if possible. Polarization studies will be conducted to further analyze the radiation. Finally, the use of a spectrometer will aid in determining the nature of the radiation that is observed.

\section{CONCLUSION}

The theory developed in this study [2] is the most complete description of off-axis undulator radiation for angles of observation greater than $1 / \gamma$. The experiment shows that coherent bunch-length-dependent radiation is observed. It is noticed that another source of radiation is present and obscures the radiation produced by the undulator. This other source of radiation is likely to be the chicane bunch compressor which produces coherent radiation that could propagate down the beam pipe. Future studies will determine the source of the radiation and will isolate the undulator radiation if possible. The bunchlength-dependent radiation that is observed is very sensitive to changes in bunch length. Small changes in bunch length produce significant changes in the output signal. Thus, the radiation that is observed is a very effective bunch length diagnostic. In fact, it has proven useful as a diagnostic when the accelerator is being tuned to compress the electron beam. The output signal gives a clear visual indication of the changes in bunch length.

\section{ACKNOWLEDGEMENTS}

The authors wish to thank John Skaritka of BNL for his help in designing the experiment, as well as George Rakowsky, Timur Shaftan, Boyzie Singh, Phil Marino, Juana Rudati, Brian Sheehy, and Jeb Berry, for their help with this experiment.

\section{REFERENCES}

[1] C.P. Neuman, W.S. Graves, and P.G. O'Shea. Phys. Rev. ST - Accel. Beams 3 (2000) 030701.

[2] C.P. Neuman. Coherent Off-Axis Undulator Radiation. Doctoral dissertation, Duke University. Durham, NC: 2001.

[3] I. Ben-Zvi et al. Proceedings of the SPIE: Free Electron Laser Challenges 2988:15-19. 